\title{
Introduction to the HICSS-50 Mobile App Development Minitrack
}

\author{
Tim A. Majchrzak \\ University of Agder, Kristiansand, Norway \\ Email: timam@uia.no
}

\author{
Tor-Morten Grønli \\ Westerdals, Oslo, Norway \\ Email: tmg@westerdals.no
}

Applications for mobile devices (apps) have facilitated the success of smartphones and tablet PCs. There are no signs of a decline of importance of app development, and new areas of app usage are about to be explored. Examples for such areas are wearables and Internet-of-Things (IoT)inspired technologies. However, the development of apps - particularly of the so called business apps - is prone to problems and companies face non-trivial technology choices.

Developing business apps poses specific requirements due to their business-critical nature. Compared to apps in general, higher quality standards should be met, apps need to be more secure and robust, and requirements need to be carefully engineered (and then met), according to industry standards. Testing of apps has been found to be very cumbersome. Whether (and to what extent) methods of classical software engineering can be applied still is matter of discussion. Moreover, challenges such as development for multiple platforms, device fragmentation, context-sensitivity, performance issues, mobility-depended security, and energy conservation arise. Therefore, new threads of research are needed to tackle these issues and pave the way for better business producibility.

The minitrack is devoted to (business) apps development and the technological background of mobile computing for corporate or other domain-specific usage. It augments the program of the Software Technology track and complements other HICSS minitracks that take a look on mobility without focussing on technology. After its successful introduction at HICSS-49 [1], we are happy to continue the minitrack also for the anniversary conference - and hopefully many more HICSS to come. For the future of the minitrack, we will seek to keep its scope up-to-date by adjusting the topic list to emerging technologies and current challenges in mobile computing.

For the second edition of the minitrack, we will have one session with four papers. These are (in order of presentation):

1) Towards a Mobile Learning Environment using Reference Architectures by Maria Lydia Fioravanti, Nemésio Freitas Duarte Filho, Lucas Bortolini Fronza, and Ellen Francine Barbosa

2) Enriching Augmented Reality with Text Data Mining: An Automated Content Management System to Develop Hybrid Media Applications by Rocco Raso, Dirk Werth, and Peter Loos
3) Exploitation and Detection of a Malicious Mobile Application by Thanh Nguyen, Jeffrey Todd McDonald, and William Bradley Glisson

4) Comprehensive Analysis of Innovative Cross-Platform App Development Frameworks by Tim A. Majchrzak, Andreas Biørn-Hansen, and Tor-Morten Grønli

We are glad that we again had many helping hands. We are proud that all authors that submitted papers to our track got at least three constructive reviews as well as an additional meta review. The average number of reviews was even four plus one meta review. We think that we outperform many journals with this effort in giving authors advice, be they accepted or not. Therefore, we would like to thank (and explicate!) our program committee for their arduous work:

- Philippe Dugerdil, Geneva School of Business Administration

- Gheorghita Ghinea, Brunel University

- Henning Heitkötter, SAP SE

- Adrian Holzer, Ecole Polytechnique Fédérale de Lausanne

- Shah Rukh Humayoun, University of Kaiserslautern

- Grace A. Lewis, Carnegie Mellon Software Engineering Institute

- Syed Atif Mehdi, University of Central Punjab

- Laura Po, University of Modena and Reggio Emilia

- Mohammad Tafiqur Rahman, University of Agder

- Christoph Rieger, University of Münster

- Sergio Ríos-Aguilar, International University of La Rioja (UNIR)

- Davide Rossi, University of Bologna

- Johannes Schobel, Ulm University

- Gabriele Taentzer, Philipps-Universität Marburg

- Tony Wasserman, Carnegie Mellon University Silicon Valley

- Burkhard Claus Wuensche, University of Auckland

- Stelios Xinogalos, University of Macedonia, Thessaloniki

\section{References}

[1] T. A. Majchrzak and H. Heitkötter, "Introduction to the mobile app development minitrack," in Proceedings 49th Hawaii International Conference on Systems Science (HICSS-49). IEEE Computer Society, 2016, p. 5682. 